\title{
Day Surgery Management of Early Breast Cancer: Feasibility and Psychological Outcomes
}

\author{
TOMMASO SUSINI ${ }^{1}$, CARLO CARRIERO $^{1}$, FRANCA TANI $^{2}$, GIULIA MATTIOLI $^{1}$, IRENE RENDA $^{1}$, \\ NICOLETTA BIGLIA ${ }^{3}$, JACOPO NORI $^{4}$, ERMANNO VANZI ${ }^{4}$ and SIMONETTA BIANCHI ${ }^{5}$ \\ ${ }^{1}$ Breast Unit, Department of Health Sciences, Gynecology Section, University of Florence, \\ Azienda Ospedaliero-Universitaria Careggi, Florence, Italy; \\ ${ }^{2}$ New Social Pathologies Research Unit, Department of Health Sciences, University of Florence, Florence, Italy; \\ ${ }^{3}$ Department of Oncological Gynecology, University of Turin, Mauriziano 'Umberto I' Hospital, Turin, Italy; \\ ${ }^{4}$ Diagnostic Senology Unit, Azienda Ospedaliero-Universitaria Careggi, Florence, Italy; \\ ${ }^{5}$ Division of Pathology, Department of Health Sciences, Azienda Ospedaliero-Universitaria Careggi, Florence, Italy
}

\begin{abstract}
Background/Aim: Breast cancer treatment represents a substantial amount of health-care costs and has a negative impact on womens' psychological health. DaySurgery managment $(D S)$ is a favorable alternative to a classic inpatient setting. In our prospective study we evaluated $D S$-treatment feasibility in terms of patient satisfaction, sameday-discharge rate, surgical-reintervention rate, psychological impact and costs. Patients and Methods: We operated on 131 early breast cancer patients in DS. Surgical outcomes were evaluated. In 64 DS-treated breast cancer patients, psychological outcomes were analyzed using validated psychometric questionnaires and comparison was made with a corresponding group of women treated as inpatients. Results: The same-day-discharge rate was $95.4 \%$. No patient required readmission. The surgical-reintervention rate was $6.2 \%$. DS-treatment significantly reduced anxiety $(p=0.05)$ and depression $(p=0.01)$ and afforded cost savings of $49 \%$. Conclusion: DS-treatment of early breast cancer was feasible, with low reintervention rate, reduced anxiety and depression, high patients' satisfaction and substantial financial savings.
\end{abstract}

Treatment for breast cancer, which is the most common female malignancy in developed countries, accounts for a substantial amount of national healthcare costs. In various countries, a heavy burden is imposed upon the health care system due to the increase in medical care costs (1), which

Correspondence to: Tommaso Susini, MD, Ph.D., Breast Unit, Department of Health Sciences, Gynecology Section, University of Florence, Azienda Ospedaliero-Universitaria Careggi, Largo Brambilla 3, 59134, Florence, Italy. Tel: +39 0552751752, e-mail: tommaso.susini@unifi.it

Key Words: Anxiety, depression, health care costs, minimallyinvasive surgical treatment, women's health. are, in part, due to the introduction of highly advanced expensive drugs and costly medical technologies. However, innovations such as the sentinel lymph node biopsy (SLNB), in association with breast-conserving surgery in early breast cancers, have substantially reduced the impact of surgical treatment, in terms of post-operative pain, need for surgical drains and patient autonomy. In conclusion, a considerable proportion of breast cancer patients may undergo quadrantectomy with SLNB and be safely discharged from the hospital on the very same day. In spite of this, in our country most patients undergoing breast conserving surgery and SLNB for treatment of early breast cancer are hospitalized for at least one or two nights. Considering that in most countries reimbursements are calculated on a fee-forservice basis, according to the diagnosis-related-group (DRG) system guidelines, it is clear that treatment for early breast cancer in a Day Surgery setting (DS) could represent a significant saving as it relates to cost of care (2).

Another relevant aspect of diagnosis and treatment for breast cancer is its dramatic negative psychological impact upon women, secondary to the fear of either dying of disease and/or impaired body image (3-5). The need for semi-private accommodations with other critical oncologic patients may further worsen psychological responses. We hypothesize that a short hospital stay for a surgical procedure with same-day discharge versus the standard two-three days inpatient hospitalization, may reduce anxiety and the depressive mood associated with diagnosis and treatment. Studies have been done to evaluate the feasibility of same-day discharge after breast cancer surgical treatment, none of which evaluated the psychological outcome of such an approach, by means of validated psychometric instruments (6).

In this prospective study, the feasibility of same-day discharge after early breast cancer surgical treatment was tested in terms of patients' satisfaction with the procedure, need for overnight stay and reoperation. A cost-benefit analysis was 
performed in order to compare DS treatment with standard inpatients' surgical management in terms of health care system costs. Furthermore, patients' outcomes were studied in terms of mood, anxiety and quality of life, by means of validated psychological testing, and compared with a control group of patients undergoing standard inpatient surgical treatment.

\section{Patients and Methods}

Patients selection. In this prospective study, we identified 131 patients with early breast cancer undergoing surgery at our Breast Unit of the Gynaecology and Obstetrics Department, Careggi Hospital, University of Florence and satisfying inclusion criteria for treatment in a DS setting. DS was defined as a surgical operation allowing patients to go home later on the very same day. Inclusion criteria were: preoperative histological diagnosis of in situ or invasive breast carcinoma with a diameter of $<3 \mathrm{~cm}$, favorable proportion between tumor and breast size allowing for breast conserving surgery, fairly good clinical conditions and low risk of lymph node metastases, meaning that both clinical examination and axillary ultrasound resulted negative. Ultrasonographic criteria for axillary lymph node evaluation are given in detail elsewhere (7-9). Patients operated in DS did not receive frozen section examination of the sentinel node. All patients gave their written informed consent. Protocol was approved by the local Ethics Committee.

Surgical procedure. The breast surgical DS procedure consisted of a wide local excision or a quadrantectomy with clear margins of at least $1 \mathrm{~cm}$ from the tumor, associated with SLNB. It was done under local anesthesia, using a mixture of Lidocaine hydrochloride $2 \%$ and Ropivacaine hydrochloride $10 \mathrm{mg} / \mathrm{ml}$. Sedation was administered (Propofol $10 \mathrm{mg} / \mathrm{ml} \mathrm{I.V.)} \mathrm{with} \mathrm{mask} \mathrm{ventilation.} \mathrm{No} \mathrm{surgical} \mathrm{drain}$ was left in situ. Ice packing was applied postoperatively during the first hour. All removed nodes were submitted for standard histological testing. In cases where sentinel lymph node macrometastases were detected at final histology, patients were readmitted mostly to undergo axillary lymph node dissection (ALND), which by contrast was not performed in cases where micrometastases or isolated tumor cells (ITC) were identified.

Questionnaries administration. We administered questionnaires to a subgroup of 64 patients with invasive breast carcinoma treated with breast-conserving surgery and SLNB, in order to rate their degree of satisfaction with the procedure and post-operative pain. The Visual Analogue Scale (VAS) (10) was used to assess pain 1 day and 7 days after the procedure. To explore the psychological impact of the procedure, in terms of mood, anxiety and depression, we administered specifically designed, validated questionnaires, one week before surgery and one month after surgery. Mood was evaluated with the 5 items "Satisfaction with Life Scale (SWLS)" (11), anxiety with the 20 items "State-Trait Anxiety Inventory" (STAI) (12) and depression with the 20 items "Center for Epidemiologic Studies Depression Scale" (CES-D) (13). We then identified a control group consisting of 64 patients matched for age, tumor stage and clinical conditions, who underwent breastconserving surgery and SLNB under general anesthesia, with traditional inpatient hospitalization for at least one post-operative night. The same questionnaries were administrated to the control group, with the same timing.
End points. End points of our study were: same-day discharge rate (defined as the percentage of patients operated on and returning home the same day of the surgical procedure), early readmission (defined as need for readmission within 48 hours from the surgical procedure) and reintervention rate (defined as the percentage of patients who needed a second surgical intervention for various reasons, i.e. axillary clearance, inadequate surgical margins, complications).

Cost assessment. By conferring with the Business Office of the hospital, the incurred costs were compiled for each DS procedure and for each traditional inpatient procedure.

Compliance with ethical standards. All procedures performed in studies involving human participants were in accordance with the ethical standards of the institutional and/or national research committee and with the 1964 Helsinki declaration and its later amendments or comparable ethical standards.

Statistical analysis. Patient characteristics were analyzed using Fisher's exact test or the Chi-square test, as appropriate. Results of the psychometric questionnaires were submitted for analysis of variance using the ANOVA test (IBM SPSS Statistics for Windows, Version 22.0. Armonk, NY: IBM Corp.) A $p$-value $<0.05$ was considered statistically significant.

\section{Results}

$D S$ feasibility. We analysed 131 patients with a mean age of 62 years. Preoperative diagnosis was in situ carcinoma in 37 cases $(28 \%)$ and invasive carcinoma in 94 cases $(72 \%)$. Overall, a wide local excision was performed in $112(85.5 \%)$ patients and a quadrantectomy in $19(14.5 \%)$ patients. In 81 of 94 women $(86 \%)$ with invasive carcinoma a SLNB was concurrently performed. Advanced age and/or relatively poor clinical conditions, were reasons for renouncing to SLNB in the remaining 13 patients. Same-day discharge was feasible in 125 of 131 patients $(95.4 \%)$. There were no patients requiring early readmission for complications. Among the remaining 6 patients, 3 were hospitalized because the operation was performed in the afternoon, although it was scheduled for the morning hours. In 5 cases, the pathological examination revealed an upgrade to invasive carcinoma initially diagnosed as ductal carcinoma in situ (DCIS), therefore we had 99 cases of invasive breast cancer (75.6\%) and 32 cases of carcinoma in situ (24.4\%). Pathological features are reported in Table I. Among the 9 positive SLN cases (macrometastases), 5 underwent subsequent ALND, 4 either refused or had comorbidities discouraging a second surgical intervention. Overall, among the 81 patients submitted for SLNB, 76 $(93.8 \%)$ did not receive any additional surgery, while 5 patients $(6.2 \%)$ underwent a second surgical procedure to perform ALND. In 2 cases $(2.4 \%)$ a widening of the breast resection margins was concurrently performed.

Questionnaries results. Between July 2016 and July 2018, we operated on 67 women with invasive breast carcinoma 
Table I. Pathological features of the 131 patients with breast carcinoma operated on in DS setting.

\begin{tabular}{lcc}
\hline pT Stage & $\mathrm{n}^{\circ}$ & $\%$ \\
\hline pTis & 32 & 24.4 \\
pT1a & 11 & 8.4 \\
pT1b & 31 & 23.7 \\
pT1c & 37 & 28.2 \\
pT2 & 8 & 6.1 \\
Total & 131 & 100.0 \\
\hline
\end{tabular}

\begin{tabular}{lcc}
\hline Histology & & \\
\hline DCIS & 28 & 21.4 \\
LCIS & 4 & 3.0 \\
IDC & 48 & 36.6 \\
ILC & 25 & 19.1 \\
Mixed & 15 & 11.4 \\
Others & 11 & 8.5 \\
Total & & \\
\hline
\end{tabular}

DCIS: Ductal carcinoma in situ; LCIS: lobular carcinoma in situ; IDC: Invasive ductal carcinoma; ILC: invasive lobular carcinoma; Mixed: IDC+ILC.

using breast conserving surgery and SLNB in DS setting. They were all invited to rate their degree of satisfaction with the procedure and to assess their emotional burden by answering questionnaires. Two of them declined; one patient was excluded because of language barrier. The remaining 64 patients were able to be evaluated for satisfaction, pain and psychological aspects. Clinical and pathological characteristics of these patients and those of the corresponding control group are presented in Table II. Data concerning pain are reported in Figure 1. According to the psychometric tests, we found a statistically significant reduction in depression and anxiety scores following DS ( $p=0.01$ and $p=0.05$, respectively) compared to women treated in a traditional inpatient setting (Figure 2).

Economic analysis. Costs associated with breast cancer DS treatment and with traditional inpatient setting were evaluated. Average cost for each DS patient was $€ 469$, compared to $€ 999$ for each one-night-inpatient case, reflecting savings of $€ 530$. The amount saved during the study period $(125 \times € 530=$ $€ 66250)$ adjusted for costs related to needed reintervention (5 $x € 999=€ 4995$ ), was $€ 61255$, corresponding to a $49 \%$ savings for treatment in DS.

\section{Discussion}

We found that surgical management of early breast cancer in DS was feasible and well accepted by most patients. Our results concerning patient satisfaction were similar to those
Table II. Clinical and pathological characteristics of the patients with invasive breast cancer treated in Day surgery (DS) and in a traditional inpatient setting (controls) and submitted for psychological testing.

\begin{tabular}{lccccc}
\hline & \multicolumn{2}{c}{ DS } & \multicolumn{2}{c}{ Controls } & \\
\cline { 2 - 4 } & $\mathrm{n}^{\circ}$ & $\%$ & $\mathrm{n}^{\circ}$ & $\%$ & $p$-Value \\
\cline { 2 - 5 } & $59(33-81)$ & - & $59(33-81)$ & - & n.s. \\
Age; Median (range) & & & & & n.s. \\
pT Stage & 10 & 15.6 & 12 & 18.8 & \\
1a & 14 & 21.9 & 14 & 21.9 & \\
1b & 32 & 50.0 & 28 & 43.7 & \\
1c & 8 & 12.5 & 10 & 15.6 & \\
2 & & & & & \\
& 64 & 100.0 & 64 & 100.0 & \\
Total & & & & & \\
& & & & & n.s. \\
Histology & 46 & 71.9 & 45 & 70.3 & \\
IDC & 10 & 15.6 & 11 & 17.2 & \\
ILC & 2 & 3.1 & 3 & 4.7 & \\
Mixed & 6 & 9.4 & 5 & 7.8 & \\
Others & & & & & \\
Total & 64 & 100.0 & 64 & 100.0 & \\
Patients with chronic pain & 2 & 3.1 & 1 & 1.6 & n.s. \\
Performance status $<80 \%$ & 4 & 6.3 & 4 & 6.3 & n.s. \\
\hline
\end{tabular}

IDC: Invasive ductal carcinoma; ILC: invasive lobular carcinoma; Mixed: IDC+ILC;

n.s.: not significant.

reported by Friedman (14) and by Goodman (15) whereas they differed from those of Marchal who reported a higher refusal rate (13.9\%) (16). In the current study, more than $95 \%$ of patients were discharged on the same day of surgery, as planned. Actually, only 3 patients $(2.3 \%)$ were not discharged for clinical reasons, while in another 3 cases, patients remained in the hospital because the surgical procedure was delayed to the afternoon hours. A different timing of these 3 operations could have therefore raised the same-day discharge rate to $97.7 \%$. Overrunning theatre lists as a cause of overnight admission have been reported also by others (17). Nevertheless, our finding is in line with other studies on DS management of breast cancer which reported same-day discharge rates ranging from $86 \%$ to $100 \%$ (1426). However, there is one small randomized study reporting lower rate of discharge on the same day of surgery $(47 \%)$ mainly due to axillary clearance following frozen section evaluation of the sentinel nodes (27). In the current series, there were no patients requiring early post-operative readmission, as also reported by others $(14-17,20,23)$, whereas some studies reported readmission rates of $6-7 \%$ due to post-op complications $(19,25)$. Pain scores did not differ between patients operated under local anesthesia with sedation in DS and those operated on traditionally, using general anesthesia. More than $96 \%$ of patients reported 

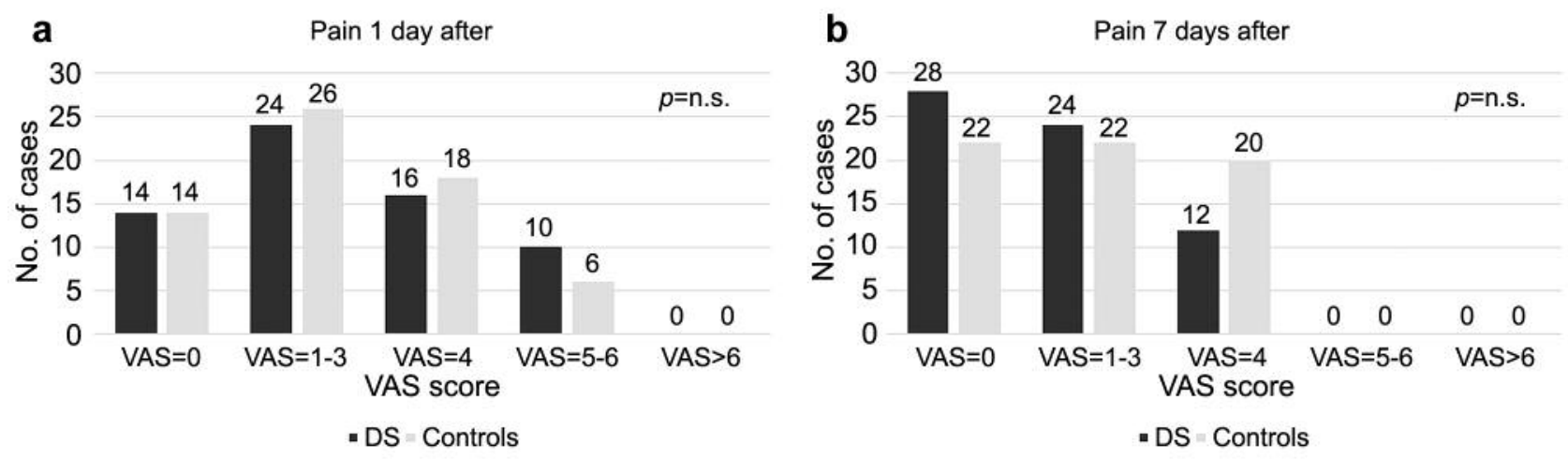

Figure 1. Pain score according to the visual analogue scale (VAS) 1 (1a) and 7 days (1b) after the surgical procedure in patients operated in Day Surgery (DS) and in a traditional inpatient setting (controls).

a

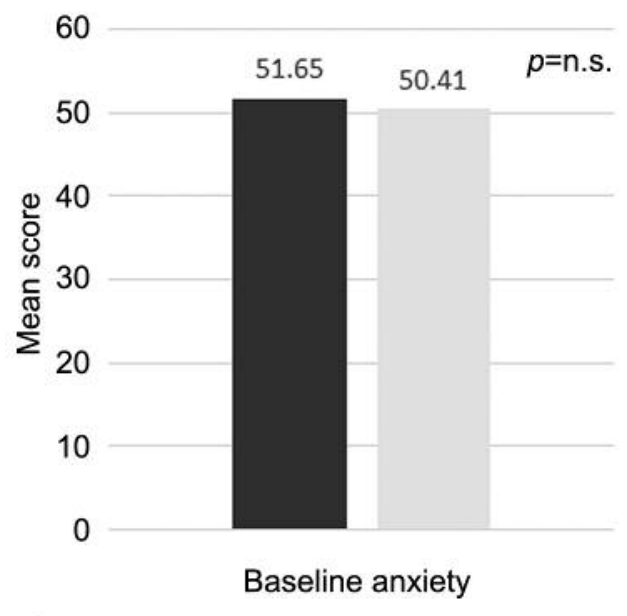

C

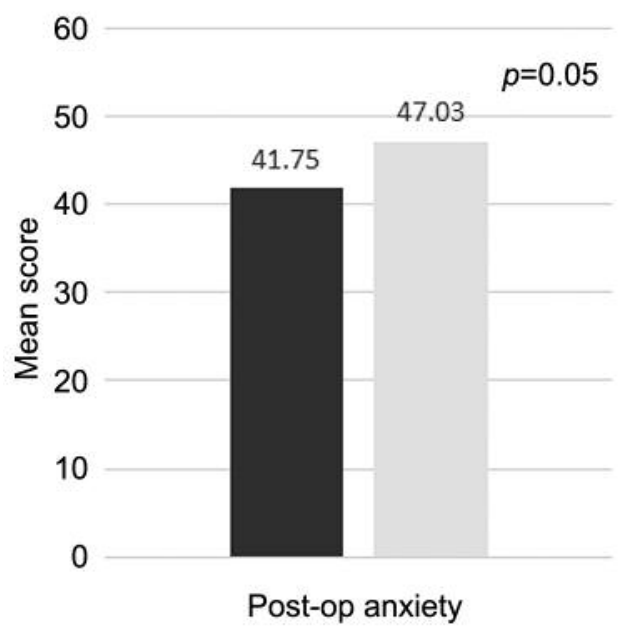

Baseline

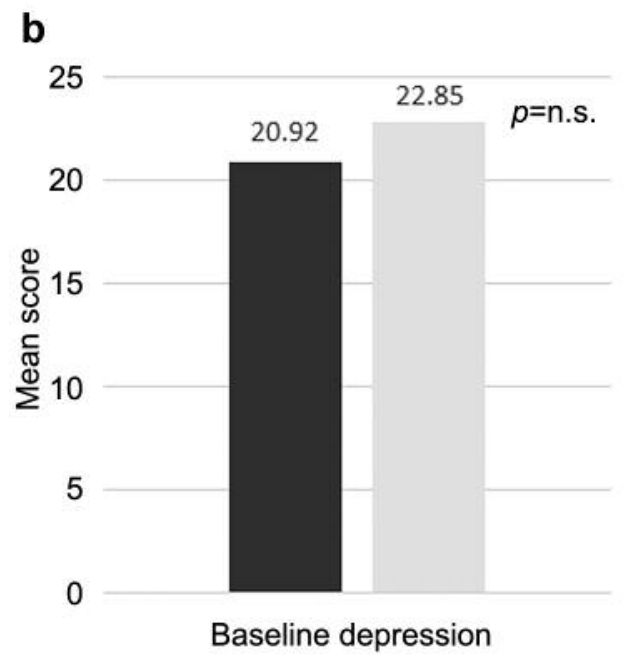

Post-operative

d

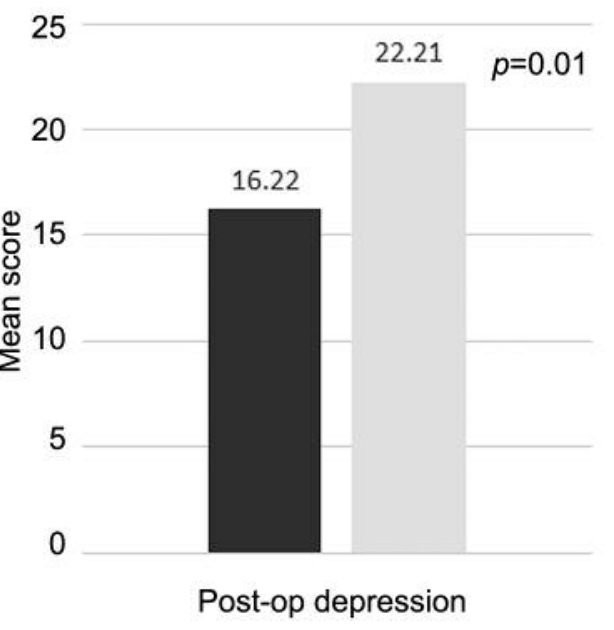

Figure 2. Baseline and post-operative mean scores of anxiety (2a; 2c), according to "State-Trait Anxiety Inventory" (STAI)(12), and depression (2b; 2d), according to the Center for Epidemiologic Studies Depression Scale (CES-D scale) (13), in women treated for breast cancer in day surgery (DS) and in a traditional inpatient setting (controls). 
positive feedback about their experience in DS. Two patients (3.1\%) were not satisfied because of excessive familial burden and anxiety related with staying at home without medical supervision during the first night. This is in line with the majority of previous reports on the topic $(14,19,20,23$, 24), with the exception of the study by Margolese, in which $40 \%$ of patients would have preferred to have spent at least one night in the hospital (28). It should be noted that a direct comparison with this latter study is not feasible as patients submitted for major breast surgery procedures such as mastectomy and ALND, were also included. Our decision of renouncing by principle to the frozen section examination of the sentinel nodes might be questioned since it is associated with a high risk of reintervention. However, the reintervention rate in the current series was only $6.2 \%$, supporting our belief that with careful pre-operative evaluation of the axillary lymph nodes by ultrasound and considering further criteria of risk for axillary node metastases $(7,9)$, it is possible to select breast cancer patients with a reasonably low risk of lymph node metastases, that may safely benefit from this minimally invasive surgical approach. Indeed, previous studies on the same subject not using axillary ultrasound in the preoperative assessment, reported much higher reintervention rates, ranging from $28-40 \%(19,22)$. After the publication of the ACOSOG Z0011 (29) and IBCSG 23-01 trials (30) and the diffusion of the American Society of Clinical Oncology clinical practice guideline updated in 2016 (31), pointing out the futility of lymphadenectomy even in the presence of metastases in the sentinel lymph node, the traditional concept of ALND in breast cancer is currently questioned (32). The utility of preoperative axillary ultrasound, as well as the possibility of avoiding ALND, are currently being evaluated in an ongoing prospective study promoted by Gentilini and Veronesi (33).

One of the most important outcomes of our study was related to psychological issues. Using validated quality-oflife-assessment tools, we obtained statistically significant lower anxiety $(p=0.05)$ and depression scores $(p=0.01)$ in patients who underwent a DS procedure in comparison with patients having an overnight stay. Indeed, patients with breast cancer have often a high grade of anxiety and depression connected with their diagnosis and surgery (3-5). Our findings support the hypothesis that early recovery in the familial environment may contribute to decreased anxiety, prevent the development of depression and feeling of helplessness related to illness. It is also possible that avoiding prolonged contact with other suffering oncologic patients may aid in reducing patients' anxiety and in preserving their positive self-image. To our knowledge, this is the first study to investigate the influence of early breast cancer treatment in a DS setting on patients' anxiety and depression using validated questionnaires.
The last relevant finding of our study was the economic outcome. Early breast cancer treatment in a DS setting was associated with savings of $49 \%$. Obviously, the cost of the operation was similar in outpatient and inpatient groups, but the further 1-2 days of hospitalization as inpatients, added an average cost of $€ 530$ per day. In the current series, even after adjusting for costs due to reintervention, breast cancer treatment in DS resulted in a potential savings of over $€ 60,000$. Our results are in line with five previous studies evaluating the economic outcomes of breast cancer treatment in DS, which resulted to reduced costs with savings ranging from $40 \%$ to $85 \%$, compared to inpatient surgery $(15,19-22)$.

In view of these several advantages, we believe that the management of early breast cancer in DS warrants encouragement. Our findings must be interpreted with caution because of the non-randomized design of the study. Moreover, psychological outcomes were evaluated in a relatively small subgroup of early breast cancer patients treated with conserving surgery and SLNB. Therefore, we cannot draw a definitive conclusion. Nonetheless, our findings of improved psychological outlooks for patients treated in DS are intriguing and further studies on a larger scale of patients are warranted.

\section{Conflicts of Interest}

All Authors declare that there is no conflict of interest regarding this study.

\section{Authors' Contributions}

TS and CC: Conceptualization, data curation, formal analysis, investigation, funding acquisition, writing original draft and editing. FT: Conceptualization, methodology, data curation, formal analysis; GM, IR and NB: Data curation, critical review, editing; JN, EV, and SB: Methodology, formal analysis, investigation.

\section{References}

1 Luengo-Fernandez R, Leal J, Gray A and Sullivan R: Economic burden of cancer across the European Union: a population-based cost analysis. Lancet Oncol 14(12): 1165-1174, 2013. PMID: 24131614. DOI: 10.1016/S1470-2045(13)70442-X

2 Jacobs VR, Bogner G, Schausberger CE, Reitsamer R and Fischer T: Relevance of health economics in breast cancer treatment: integration of economics in the management of breast cancer at the clinic level. Breast Care 8(1): 7-14, 2013. PMID: 24715837. DOI: $10.1159 / 000348370$

3 Schain W, d'Angelo T, Dunn M, Lichter A and Pierce L: Mastectomy versus conservative surgery and radiation therapy. Psychosocial consequences. Cancer 73(4): 1221-1228, 1994. PMID: 8313326.

4 Ganz PA, Rowland JH, Desmond KA, Meyerowitz BE and Wyatt GE: Life after breast cancer: Understanding women's health-related quality of life and sexual functioning. J Clin Oncol 16(2): 501-514, 1998. PMID: 9469334. DOI: 10.1200/ JCO.1998.16.2.501 
5 Avis NE, Crawford S and Manuel J: Quality of life among younger women with breast cancer. J Clin Oncol 23(15): 33223330, 2005. PMID: 15908646. DOI: 10.1200/JCO.2005.05.130

6 Marla S and Stallard S: Systematic review of day surgery for breast cancer. Int J Surg 7(4): 318-323, 2009. PMID: 19427922. DOI: $10.1016 /$ j.ijsu.2009.04.015

7 Susini T, Nori J, Olivieri S, Molino C, Marini G, Bianchi S, Vezzosi V, Livi L, Mascalchi M and Scarselli G: Predicting the status of axillary lymph nodes in breast cancer: a multiparameter approach including axillary ultrasound scanning. Breast 18(2): 103108, 2009. PMID: 19285396. DOI: 10.1016/j.breast.2009. 02.001

8 Susini T, Nori J, Vanzi E, Livi L, Pecchioni S, Bazzocchi M, Mangialavori G, Branconi F and Scarselli G: Axillary ultrasound scan in the follow up of breast cancer patients submitted to sentinel node biopsy. Breast 16(2): 190-196, 2007. PMID: 17140793. DOI: 10.1016/j.breast.2006.10.004

9 Nori J, Vanzi E, Bazzocchi M, Bufalini FN, Distante V, Branconi $\mathrm{F}$ and Susini T: Role of axillary ultrasound examination in the selection of breast cancer patients for sentinel node biopsy. Am J Surg 193(1): 16-20, 2007. PMID: 17188081. DOI: 10.1016/ j.amjsurg.2006.02.021

10 Reips UD and Funke F: Interval level measurement with visual analogue scales in internet-based research: "VAS Generator". Behav Res Methods 40(3): 699-704, 2008. PMID: 18697664.

11 Pavot W and Diener E: Review of the satisfaction with life Scale. Psychol Assess 5(2): 164-172, 1993. DOI: 10.1037/10403590.5.2.164.

12 Spielberger CD, Gorsuch RL, Lushene R, Vagg PR and Jacobs GA: Manual for the State-Trait Anxiety Inventory. Palo Alto, CA: Consulting Psychologists Press, 1983.

13 Eaton WW, Muntaner C, Smith C, Ybarra M and Tien A: Center for epidemiologic studies depression scale: Review and revision (CESD and CESD-R). In: Maruish ME (ed.). Mahwah, NJ, US Lawrence Erlbaum Associates Publishers, pp. 363-377, 2004.

14 Friedman D, Gianetta E, Giaminardi E, Aicardi M and Bachi V: Definitive breast cancer surgery as an outpatient: rationale and our experience. Ann Ital Chir 75(5): 525-528 (discussion 529), 2004. PMID: 15960338.

15 Goodman AA and Mendez AL: Definitive surgery for breast cancer performed on an outpatient basis. Arch Surg 128(10): 1149-1152, 1993. PMID: 8215874.

16 Marchal F, Dravet F, Classe JM, Campion L, François T, Labbe D, Robard S, Théard JL and Pioud R: Postoperative care and patient satisfaction after ambulatory surgery for breast cancer patients. Eur J Surg Oncol 31(5): 495-499, 2005. PMID: 15922885. DOI: 10.1016/j.ejso.2005.01.014

17 Athey N, Gilliam AD, Sinha P, Kurup VJ, Hennessey C and Leaper DJ: Day-case breast cancer axillary surgery. Ann R Coll Surg Engl 87(2): 96-98, 2005. PMID: 15826416. DOI: $10.1308 / 1478708051667$

18 Seltzer MH: Partial mastectomy and limited axillary dissection performed as a same day surgical procedure in the treatment of breast cancer. Int Surg 80(1): 79-81, 1995. PMID: 7657499.

19 Carcano G, Uccella L, Ferrari A, Rovera F, Dionigi G, Limonta $\mathrm{G}$ and Dionigi R: Breast cancer surgery as an outpatient in Italy: is it possible? Chirurgia 18(5): 323-326, 2005.

20 Dooley WC: Ambulatory mastectomy. Am J Surg 184(6): 545548, 2002. PMID: 12488162.

21 McManus SA, Top DA and Hopkins C: Advantages of outpatient breast surgery. Am Surg 60(12): 967-970, 1994. PMID: 7992976.
22 De Kok M, Dirksen CD, Kessels AG, van der Weijden T, van de Velde CJ, Roukema JA, Bell AV, van der Ent FW and von Meyenfeldt MF: Cost-effectiveness of a short stay admission programme for breast cancer surgery. Acta Oncol 49(3): 338-346, 2010. PMID: 20397768. DOI: 10.3109/028418610036 10192

23 Marrazzo A, Taormina P, David M, Lo Gerfo D, Casà L, Noto A and Mercadante S: Surgical treatment of early breast cancer in day surgery. Chir Ital 59(5): 687-691, 2007. PMID: 18019641.

24 Guinaudeau F, Beurrier F, Rosay H, Carrabin N, Faure C, Ferraioli D and Chopin N: Satisfaction in ambulatory patients with breast-conserving surgery and sentinel node biopsy for breast cancer. Gynecol Obstet Fertil 43(3): 213-218, 2015. PMID: 25749187. DOI: 10.1016/j.gyobfe.2015.01.013

25 Dravet F, Belloin J, Dupre PF, François T, Robard S, Theard JL and Classe JM: Role of outpatient surgery in breast surgery. Prospective feasibility study. Ann Chir 125(7): 668-676, 2000. PMID: 11051698.

$26 \mathrm{Ng} \mathrm{YY,} \mathrm{Chan} \mathrm{PM,} \mathrm{Chen} \mathrm{JJ,} \mathrm{Seah} \mathrm{MD,} \mathrm{Teo} \mathrm{C} \mathrm{and} \mathrm{Tan} \mathrm{EY:}$ Adopting ambulatory breast cancer surgery as the standard of care in an Asian population. Int J Breast Cancer 2014: 672743, 2014. PMID25197577. DOI:10.1155/2014/672743

27 Ojala K, Vironen JH, Mattila K, Eklund AM, Leidenius MH and Meretoja TJ: Feasibility of day surgery in patients with breast conservation and sentinel node biopsy: a randomized controlled trial. Scand J Surg 105(1): 29-34, 2016. PMID: 25922474. DOI: 10.1177/1457496915583201

28 Margolese RG and Lasry JC: Ambulatory surgery for breast cancer patients. Ann Surg Oncol 7(3): 181-187, 2000. PMID: 10791847.

29 Giuliano AE, Hunt KK, Ballman KV, Beitsch PD, Whitworth PW, Blumencranz PW, Leitch AM, Saha S, McCall LM and Morrow M: Axillary dissection vs no axillary dissection in women with invasive breast cancer and sentinel node metastasis: a randomized clinical trial. JAMA 305(6): 569-575, 2011. PMID: 21304082. DOI: 10.1001/jama.2011.90

30 Galimberti V, Cole BF, Zurrida S, Viale G, Luini A, Veronesi P, Baratella P, Chifu C, Sargenti M, Intra M, Gentilini O, Mastropasqua MG, Mazzarol G, Massarut S, Garbay JR, Zgajnar J, Galatius H, Recalcati A, Littlejohn D, Bamert M, Colleoni M, Price KN, Regan MM, Goldhirsch A, Coates AS, Gelber RD and Veronesi U: Axillary dissection versus no axillary dissection in patients with sentinel-node micro metastases (IBCSG 23-01): a phase 3 randomized controlled trial. Lancet Oncol 14(4): 297-305, 2013. PMID: 23491275. DOI: 10.1016/S1470-2045(13)700 35-4

31 Lyman GH, Somerfield MR and Giuliano AE: Sentinel lymph node biopsy for patients with early-stage breast cancer: 2016 American Society of Clinical Oncology clinical practice guideline update summary. J Oncol Pract 13(3): 196-198, 2017. PMID: 28118104. DOI: 10.1200/JOP.2016.019992

32 Galimberti V, Corso G, Monti S and Pagani G: Over exploring and overtreating the axilla. Breast 31: 290-294, 2017. PMID: 27282983. DOI: $10.1016 /$ j.breast.2016.05.002

33 Gentilini $\mathrm{O}$ and Veronesi U: Abandoning sentinel lymph node biopsy in early breast cancer? A new trial in progress at the European Institute of Oncology of Milan (SOUND: Sentinel node vs Observation after axillary Ultrasound). Breast 21(5): 678-681, 2012. PMID: 22835916. DOI: 10.1016/j.breast.2012. 06.013

Received May 8, 2019

Revised May 22, 2019 Accepted May 23, 2019 